\title{
Evaluation of Causes and Impacts of Emotional Pressure Among Teenagers
}

\author{
Che Jiayao \\ College of education science, Minnan Normal University, Zhangzhou, 363000, China \\ *Corresponding author. Email: cjy07212021@outlook.com
}

\begin{abstract}
This study assessed how teenagers reach a point of emotional stress which eventually results in anxiety and depression among the young generation within the society. Particularly, the general theme of this study is built upon the notion that emotions have been shown to impose substantial negative impact on teenagers. This paper employed a descriptive research methodology in which pieces of literature are employed in providing evidence to the key thematic concerns. In this regard, this study attempted to attest to the fact that an apt understanding of the causes and impacts of emotional pressure among teenagers is substantively important in the development and maintenance of emotional stability, besides proper social habits that support the mental wellbeing of teenagers. Thus, this study finds that the management program implemented among teenagers is rather different from that adopted by adults. Moreover, the results revealed that negative emotions among teenagers largely result from the aspects that make up their social environment, including bullying, poor parenting, and or sexual violence. More importantly, this study points out that negative emotions have rusted in mood disorders, insomnia, social-anxiety disorders and several depressive disorders. This research demonstrates that the Public is tasked with the obligation of correcting such a problem among teenagers since it may become a long-term issue as the same group of teenagers join adulthood.
\end{abstract}

Keywords: Emotion pressure, Mood disorders, Sleep deficiencies, Social anxiety disorder.

\section{INTRODUCTION}

Emotional pressure, also known as emotional stress, is one of the greatest menaces facing teenagers in the twenty-first century. Several reports published within the past decade have indicated high levels of stress among teens than adults. Accordingly, teens have repeatedly recorded poor mental health, stimulated by upward spiraling levels of depression and anxiety. In the United States, the average score for stress among teens is rated at 5.8 out of 10 compared to 3.8 out of 10 for adults [1]. In Japan, research indicates that teen suicide is three times higher today than any other time in history. According to a report from Agence FrancePresse, an average of five hundred Japanese students aged twenty years and below commits suicide each year [2]. Also, more than 10.6 percent of Chinese adolescents have recorded high levels of depressive symptoms, indicating a prevalence of emotional pressure [3]. Therefore, emotional pressure is among the leading cause of mental illness among teenagers in our contemporary society.
Teenage marks a period of increased risk of the beginning of the depression and anxiety disorders[6]. Evidence suggests that childhood adversity and stressful life occurrences are huge risk factors regarding future psychopathology [4]. Moreover, it is suggested that the ability to control emotional reactions to such occurrences may serve a mediating role. With the increased novel demands and independence during teenage as compared to childhood, teenagers may find it essential to control their emotions in line with the nature of their stressors. Failure to act so may heighten the risk of mental health complications. This study aims to understand the causes of emotional stress among teenagers and more importantly, its impact on their overall development. This study employs a structural literature review technique in which several studies are used to shine a light on the thematic explanations. In this context, this study is motivated by the need to emphasize the need for emotional regulation among teenagers in response to the relevant stressors. To achieve this objective, this study will be divided into three sections. To begin with, the first section addresses the causes of emotional stress among teenagers. 
Subsequently, the next section will address the impacts of emotional stress on teenagers before the appropriate inferences and conclusions are made.

\section{CAUSES OF EMOTIONAL STRESS AMONG TEENAGERS.}

Emotional stress can be categorized into teenage stress and adult stress[5]. The classification is based on signs and symptoms that individuals manifest when they are subjected to stress. The management program for dealing with teen stress is different from that of adult stress because the causes of the two are different. As an intermediate group between childhood and adulthood, teenagers are exposed to a tedious decision-making environment. They are supposed to deal with educational demands and frustrations, family feuds and wrangles, and changes in their bodies. Consequently, this group is rendered vulnerable to physical and emotional stress. According to a report from the Centers for Disease Control and Prevention on Children's mental health, "teenagers suffer at least one episode of stress every week." Further, Kachroo's research [5], indicates that school work is responsible for 78 percent of stress among teenagers, romantic relationships, siblings, and problems with friends, all come second with 64 percent. Other causes include family responsibilities, divorce or separation of parents, financial challenges, negative thoughts and feelings, and changing relationships with peers.

\subsection{Academic pressure}

According to Sun "Academic pressure is a major risk factor for poor mental health and suicide and other harmful behaviors" among teenagers. In China, Education is among the major cause of stress among teenagers [3]. This is backed up by the rising number of suicide cases connect to poor academic performance, especially among high school students. According to the People's Daily Onlinea 12th -grade boy in Guangdong Province attempted to commit suicide due to failure in exams and cyber love. A similar case was recorded in Hubei Province the same year whereby a 16-year-old tried to take his life, citing poor academic grades as the reason . Ang, Huan, and Braman argue that Students in China and other parts of East Asia experience a heavier education burden compared to their counterparts in Western countries [7]. As a result, emotional stress due to academic pressure is high in China. In the US, depression, anxiety, and behavioral disorders have been high among children between the ages of 11 to 15 years. According to the CDC (n.d.), "7.4\% of children ages 317 years (approximately 4.5 million) have a diagnosed behavior problem." Over 4 million children within the same age have been diagnosed with anxiety, and 1.9 million suffer from depression. Bouchrika submits that 75 percent of high school students in the US always feel stressed by schoolwork[1]. Other researchers have also concluded that education is a major source of stress to teenagers due to the high expectations from their parents in terms of academic performance and competition between students. All these subject teenagers to emotional pressure, especially when parents castigate their children for failing in school.

\subsection{Romantic relationships}

Apart from academic demands and frustrations, another major cause of emotional pressure among teenagers is romantic relationships, better known as 'young love.' research conducted by Anderson, Salk, and Hyde shows that there is a direct relationship between stress levels and romantic relationships among teenagers [20]. As children in this group start discovering their body framework and functioning, most tend to indulge in romantic relationships with partners they are attracted to. The dating process during this stage has been associated with several adolescent depressive symptoms such as sleep changes, mood swings, irritability, deviant behavior, and social exclusion. In research to establish the impact of 'young love' on teenagers, Price et al. discovered that the rate of maintaining and sustaining romantic relationships among teenagers is very low, and in most cases, the breakups lead to self-harm, suicide attempts, and significant disruption of adolescents' mental health. In the US, over 50 percent of teenagers have dated by the age of 15 (CDC, n.d.) [10]. Most of these relationships are labeled as "depressogenic," and according to Steinberg and Davila, a romantic breakup among teenagers "...increases chances for a first major depressive episode in adolescence" [11]. Another important consideration, in this case, is that boys and girls approach teenage romantic relationships differently; therefore, self-harm, suicide, and mental health tend to vary by gender [10]. For instance, girls matured faster than boys and are more likely to engage in romantic relationships, especially with older people (in their twenties) than them. Consequently, they become vulnerable and may easily become victims of post-relationship stressors. On the other hand, boys tend to experience adolescence at a relatively late stage. For instance, Anderson, Salk, and Hyde record that by the age of 13 , girls who have undergone early maturity are likely to be in a romantic relationship while boys, especially those who experience late maturity, might happen at 18 years. As a result, "...the higher levels of romantic stress at age 15 would be associated with greater depressive symptoms concurrently at age 15 and prospectively at age 18 in both boys and girls" [20].

\subsection{Negative Parenting Approach}

Additionally, emotional stress among teenagers can result from negative parenting styles, rejection, or 
separation. Huo et al. indicate that parental rejection or overprotection in China is among the leading causes of mild depression and anxiety. Parenting style also correlated moderately with depression and mildly with anxiety[12]. Additionally, the research indicated that boys and girls react variedly to parental rejection or parenting style. According to $\mathrm{Xu}$ et al. and Stevens, parenting style can be either positive or negative. A positive parenting style is perceived as warm and supportive, while a negative parenting style is characterized by overprotection, coercive interactions, and harshness $[13,14]$. and Rodriguez et al. record that adolescents are more likely to manifest depression and anxiety disorders for many reasons, but the primary one being that they are highly responsive to their environment[15]. For example, in the US, teenagers with anxiety symptoms are more likely to have authoritarian parents. Separation is also a leading cause of anxiety and restlessness. According to Bouchrika , "over 30\% of American college students feel homesick. Freshmen take it even worse, as $69 \%$ reported a feeling of severe homesickness [1]." In Germany, a survey has shown that depression and anxiety resulting from negative parenting styles are likely to have a long-term impact on a teenager's life. Therefore, family is an important part of teenagers' mental health, and its absence can easily lead to deviant behaviors such as drug abuse, premature sexual indulgence, crime, among other social ills.

\subsection{Bullying and Harassment}

Other causes of emotional stress include bullying and harassment from siblings or friends. Family, especially, parents play a critical role in determining their children's mental states and providing them with the necessary guidance. Nonetheless, separated parents or parents who have rejected their child cannot detect whether they are being bullied in school. In the US, 29 percent of college students are victims of bullying, while 37 percent of middle and high school students have experienced bullying, particularly cyberbullying. It is crucial to note that bullying and harassment among boys hold different impacts on girls. Research reveals that the likelihood of bullying and harassment for female and male students in the US is 24 percent to 17 percent. Bullying may include; physical bullying, exclusion from activities on purpose, and subjecting some students to vicious rumors. The result of these activities is a disrupted mental condition, whereby the victims may experience low self-esteem, depression, suicidal thoughts, and social aversiveness.

\section{IMPACTS OF EMOTIONAL STRESS ON TEENAGERS}

Stress affects physical, psychological, and social development among teenagers. It introduces adolescents to psychological disorders such as depression and anxiety. Given that teenage is a critical period for brain maturation, altering the normal mental health of teenagers subjects them to slow brain development or chronic disruption of structural maturation. Further, emotional pressure lays teens open to drug and substance abuse, academic failure, mood disorders, and disrupted sleep patterns.

\subsection{Mood Disorders}

Research in psychology has revealed a direct correlation between emotional stress and mood disorders among teens. Lack and Green argue that bipolar disorder, major depression, and dysthymia are among the major mood disorders exhibited among adolescents[16]. These disorders can lead to impairment across various areas of functioning, including social and academic arenas. Based on DSM-IV-TR Mood Disorders, mood disorders can be categorized into "depressive disorders, bipolar disorders, mood disorder due to medical condition, and substance-induced mood disorders" [16]. Four models explain the sources of mood disorders; the biological model, cognitive model, behavioral model, and interpersonal model. According to the interpersonal model, mood disorders occur due to a lack of trust and love from close to an individual. For instance, if a parent rejects a child or is too harsh towards their child, they may easily slide into depression[8]. Therefore, a constant negative interaction between parents and adolescents is likely to precipitate four types of mood episodes: major depressive, maniac, mixed, and hypomanic. The difference between the presence of these disorders among children and adults is the degree to which they interfere with an individual's normal functioning [20]. For instance, while adults are able to withstand high levels of negative interaction from their environment, for adolescents, this can be a catalyst to one's self-harm, negative self-perception, among other depressive symptoms.

Major depressive disorders (MDD) occur as a result of having more than one depressive disorder. In this case, teenagers present with disruptive behaviors exuded both psychologically and physically. For one to be diagnosed with MDD, they have to present with the following symptoms every day within two weeks [16]. First, they should be depressed for a better part of the day; second, they should show a lack of interest in all activities; third, they should exhibit disrupted appetite; fourth, they should present with fatigue most of the time; fifth, they should present with feelings of worthlessness, sixth, they should show an inability to think or concentrate on anything, and lastly, they should exhibit suicidal ideation or attempts. Consequently, such teens cry most of the time, suffer from weight loss, and exhibit repeated emotional outbursts. Other effects of MDD include; indifference, lack of cooperation, and 
somatic complaints like aching arms, headaches, and stomachaches.

Another mood disorder is the dysthymic disorder presented by the DSM-IV-TR as a depressive disorder that subjects adolescents to an irritable mood that persists for at least twelve months. It is characterized by sleep disturbance, hopelessness, lack of appetite, fatigue, and inability to concentrate [16]. Diagnosis of dysthymia can be made when an adolescent presents with the aforementioned symptoms, dysthymia is a lowgrade depressive disorder with less severity compared to major depressive disorder[16].

Like dysthymic disorder, bipolar disorder is also a low-grade mood disorder characterized by mood swings ranging from extremely low to mania depression. According to Lack and Green, "bipolar disorder in adolescents is a controversial topic due to the many diagnostic obstacles present" [16]. For instance, misinterpretation of symptoms is one of the obstacles that make the diagnosis of bipolar disorder among adolescents a cautious expedition. Teens might present with mood swings, irritability, and defiance, but that does not qualify them as victims of bipolar disorder. Therefore, it requires highly experience clinicians to diagnose bipolar disorder, lest a wrong diagnosis can be made. DSM-IV-TR classifies bipolar disorders into Bipolar I and Bipolar II. The difference between the two is that Mixed or manic depressive episodes characterize bipolar while Bipolar II shows hypomanic episodes [17]

\subsection{Sleep Deficiencies}

Researchers have explored sleep patterns and rates of insomnia among adolescents, aiming to establish their cause and impact on the teens' mental health and structural maturation. There is a general agreement among scholars' findings that emotional pressure is a major trigger of disruptive sleep patterns. According to research conducted among adolescents between 16-18 years in Hordaland County, Norway, it was reported that 65 percent of adolescents experience "sleep onset latency exceeding 30 minutes" [18]Further, "an American population-based study reported a $10.7 \%$ lifetime prevalence of insomnia according to the DSMIV criteria" (p. 2). The lifetime prevalence of insomnia occurs as a result of extreme insomnia experienced during teenage. Poor sleep patterns and insomnia among teens develop due to rejection from parents, academic pressure, and trauma from bullying experiences, among others [23]. According to Zhou et al., “...prevalence of insomnia symptoms in Chinese adolescents ranges from $3.4 \%$ to $12.2 \%$." Most students extend over the night for studies or smartphone use[22]. The general effect of this cycle is a growing destabilization of teenagers' emotional stability, cognitive functioning, and memory disruption. In Japan, depression and suicidal ideation among teens have been associated with stress and insufficient sleep. In research to establish how emotional pressure affects school performance, Rhie and Chae concluded that students who slept for long hours were able to perform well compared to their counterparts who experienced insomnia [19].

\subsection{Social Anxiety Disorder}

The National Institute of Mental Health defines social anxiety as "persistent fear of one or more social or performance situations in which the person is exposed to unfamiliar people or possible scrutiny by others [9]." A person suffering from social anxiety tends to act in a humiliating or embarrassing way due to lack of confidence. 9.1 percent of children aged between 13 to 18 years are likely to suffer from social anxiety disorder with $1.3 \%$ percent presenting with severe cases in the US. In research to determine impairment among adolescents using the DSM-IV criteria, it was found that females experienced high levels of social anxiety, 11.2 percent, compared to 7 percent, among males [9]. Social anxiety occurs as a result of negative experiences such as neglect from parents, bullying, and sexual violence. It is common among teenagers, with reports indicating that one out of 3 teenagers suffers from social anxiety. In the US, over 19 million people suffer from social anxiety. Additionally, social anxiety has been identified as the most common anxiety disorder and the third most common mental health disorder in the US [21].

\section{CONCLUSION}

This study assesses and understands the causes and effects of emotional stress on adolescents, especially the development of adolescents.It is understood that several factors may lead to emotional stress including academic stress and romantic relationships which may pile pressure on an individual to exhibit a consequence of the same. The latter may point to social anxiety disorder, sleep deficiencies and mood disorders.

Generally, the impacts of emotional pressure among teenagers in contemporary society are a subject of attention because they breed more social ills than virtues. Therefore, it is imperative to gain a deeper understanding of what causes emotional pressure among teenagers in order to develop an effective counteractive model. Moreover, scholars have emphasized the essence of developing and maintaining emotional and social habits that promote mental well-being among teenagers. At this formative stage, the foundations to adulthood life are laid, requiring meticulous observance of a teenagers' social, psychological, and physical changes and how that affects their structural maturity.

\section{ACKNOWLEDGMENT}

Firstly, I would like to show my deepest gratitude to my teachers and professors, who have teached me 
professional knowledge and writing experience and provided me with valuable guidance in every stage of the writing of this thesis. Thank you very much for their valuable suggestions. I have felt the rigorous academic attitude from my teachers and also found my own deficiency. Further, I would like to thank all my friends and parents for their encouragement and support. Without all their enlightening instruction and impressive kindness, I could not have completed my thesis. This period will be a precious memory in my life.

\section{REFERENCES}

[1] Bouchrika, I. (2020). 50 Current Student Stress Statistics: 2020/2021 Data, Analysis \& Predictions « Guide 2 Research. Guide2research.com. Retrieved 24 July 2021, from https://www.guide2research.com/research/studentstress-statistics.

[2] Agence France-Presse (2018). Japanese schools on suicide watch as students return after holidays. SCMP.

[3] Sun, J., Dunne, M. P., \& Hou, X. Y. (2012). Academic stress among adolescents in China. Australasian Epidemiologist, 19(1), 9-12.

[4] McLaughlin, K. A. (2016). Future directions in childhood adversity and youth psychopathology. Journal of Clinical Child \& Adolescent Psychology, 45(3), 361-382.

[5] Kachroo, W. Q. (2014). Stress among adolescents in Jammu and Kashmir. International Journal in Management \& Social Science, 2(3), 47-60.

[6] Rapee, R. M., Kennedy, S., Ingram, M., Edwards, S., \& Sweeney, L. (2005). Prevention and early intervention of anxiety disorders in inhibited preschool children. Journal of consulting and clinical psychology, 73(3), 488.

[7] Ang, R. P., Huan, V. S., \& Braman, O. R. (2007). Factorial structure and invariance of the Academic Expectations Stress Inventory across Hispanic and Chinese adolescent samples. Child psychiatry and human development, 38(1), 73-87.

[8] Mendelson T. (2013) Stress, Emotional. In: Gellman M.D., Turner J.R. (eds) Encyclopedia of Behavioral Medicine. Springer, New York, NY. https://doi.org/10.1007/978-1-4419-1005-9_289

[9] NIMH. (n.d.) Social Anxiety Disorder. Nimh.nih.gov. (2021). Retrieved 24 July 2021, from

https://www.nimh.nih.gov/health/statistics/socialanxiety-disorder.
[10] Price, M., Hides, L., Cockshaw, W., Staneva, A. A., \& Stoyanov, S. R. (2016). Young love: Romantic concerns and associated mental health issues among adolescent help-seekers. Behavioral Sciences, 6(2), 9.

[11] Steinberg, S. J., \& Davila, J. (2008). Romantic functioning and depressive symptoms among early adolescent girls: The moderating role of parental emotional availability. Journal of Clinical Child \& Adolescent Psychology, 37(2), 350-362.

[12] Hou, Y., Xiao, R., Yang, X., Chen, Y., Peng, F., Zhou, S., ... \& Zhang, X. (2020). Parenting style and emotional distress among Chinese college students: A potential mediating role of the Zhongyong thinking style. Frontiers in Psychology, 11.

[13] Xue, Y., Li, Q., Jin, L., Feng, L., Clifton, D. A., \& Clifford, G. D. (2014, April). Detecting adolescent psychological pressures from micro-blog. In International Conference on Health Information Science (pp. 83-94). Springer, Cham.

[14] Stevens A. E. (2014). Negative Parenting in Childhood Differentially Affects the Adjustment of College Students with and Without ADHD. Master' $\mathrm{s}$ thesis, Appalachian State University, Boone, NC

[15] Rodriguez C. M., Tucker M. C., Palmer K. (2016). Emotion regulation in relation to emerging adults' mental health and delinquency: a multiinformant approach. J. Child Fam. Stud. 251916 1925. 10.1007/s10826-015-0349-6

[16] Lack, C. W., \& Green, A. L. (2009). Mood disorders in children and adolescents. Journal of pediatric nursing, 24(1), 13 - 25. https://doi.org/10.1016/j.pedn.2008.04.007

[17] Apps, J., Winkler, J., \& Jandrisevits, M. D. (2008). Bipolar disorders: symptoms and treatment in children and adolescents. Pediatric nursing, 34(1), $84-88$.

[18] Hysing, M., Pallesen, S., Stormark, K. M., Lundervold, A. J., \& Sivertsen, B. (2013). Sleep patterns and insomnia among adolescents: a population - based study. Journal of sleep research, 22(5), 549-556.

[19] Rhie, S., \& Chae, K. Y. (2018). Effects of school time on sleep duration and sleepiness in adolescents. PloS one, 13(9), e0203318.

[20] Anderson, S. F., Salk, R. H., \& Hyde, J. S. (2015). Stress in romantic relationships and adolescent depressive symptoms: Influence of parental 
support. Journal of Family Psychology, 29(3), 339 - 348. https://doi.org/10.1037/fam0000089

[21] Keeley, M. L., \& Storch, E. A. (2009). Anxiety disorders in youth. Journal of Pediatric Nursing, 24(1), 26-40.

[22] Zhou, S. J., Wang, L. L., Yang, R., Yang, X. J., Zhang, L. G., Guo, Z. C., \& Chen, J. X. (2020). Sleep problems among Chinese adolescents and young adults during the coronavirus-2019 pandemic. Sleep medicine, 74, 39-47

[23] Kang, V., Shao, J., Zhang, K., Mulvey, M., Ming, X., \& Wagner, G. C. (2012). Sleep deficiency and sleep health problems in chinese adolescents. Clinical Medicine Insights: Pediatrics, 6, CMPed-S8407. 